\title{
Clinical Study \\ Intravitreal Dexamethasone in the Management of Delayed-Onset Bleb-Associated Endophthalmitis
}

\author{
David J. Jacobs, ${ }^{1}$ Avinash Pathengay, ${ }^{1}$ Harry W. Flynn Jr., ${ }^{1}$ Theodore Leng, ${ }^{2}$ \\ Darlene Miller, ${ }^{1}$ and Wei Shi ${ }^{1}$ \\ ${ }^{1}$ Department of Ophthalmology, Bascom Palmer Eye Institute, Miller School of Medicine, University of Miami, \\ Miami, FL 33136, USA \\ ${ }^{2}$ Department of Ophthalmology, Byers Eye Institute at Stanford, Stanford School of Medicine, Palo Alto, CA 94303, USA
}

Correspondence should be addressed to Harry W. Flynn Jr., hflynn@med.miami.edu

Received 7 August 2011; Accepted 23 October 2011

Academic Editor: Meredith Gregory-Ksander

Copyright () 2012 David J. Jacobs et al. This is an open access article distributed under the Creative Commons Attribution License, which permits unrestricted use, distribution, and reproduction in any medium, provided the original work is properly cited.

\begin{abstract}
Purpose. To report the visual acuity (VA) outcomes and culture results of delayed-onset bleb-associated endophthalmitis (BAE) with and without intravitreal dexamethasone (IVD). Methods. Retrospective nonrandomized comparative case series of BAE at Bascom Palmer Eye Institute between January 1, 1996 and December 31, 2009. Clinical data were compared using the 2-sided Student's $t$-test for patients who received IVD and patients who did not receive IVD. Results. 70/83 (84\%) received IVD, and $13 / 83(16 \%)$ did not receive IVD. Mean baseline VA was 20/90 in the IVD group and 20/70 in the group that did not receive IVD $(P=0.57)$. Mean presenting VA was $0.9 / 200$ in the IVD group and $1.7 / 200$ in the group that did not receive IVD $(P=0.23)$. Repeat cultures were positive in $2 / 70(3 \%)$ IVD cases and $1 / 13(8 \%)$ cases that did not receive IVD $(P=0.57)$. Mean VA at 1 month was $5 / 200$ in the IVD group and $1.8 / 200$ in the group that did not receive IVD, $\log$ MAR $\Delta$ of 0.85 and 1.56 , respectively $(P=0.02)$. Mean VA at 3 months was $7 / 200$ in the IVD group and $3 / 200$ in the group that did not receive IVD, $\operatorname{logMAR} \Delta$ of 0.74 and 1.33 , respectively $(P=0.14)$. Conclusion. In the current study of BAE, IVD was associated with improved short-term VA outcomes without an increased rate of persistent infection.
\end{abstract}

\section{Introduction}

Since 1974 intravitreal dexamethasone (IVD) has been used as an adjunct to intravitreal antibiotics in the management of bacterial endophthalmitis [1-3]. In 1992, Irvine et al. reported favorable outcomes in a series of Gram-negative endophthalmitis cases treated with adjunctive IVD [2]. In $200443 \%$ of retina specialists responded that they use IVD in the management of postcataract endophthalmitis [4]. The role of IVD in the management of bacterial endophthalmitis remains controversial due to contradictory results reported by small, comparative studies [5-8].

In delayed-onset bleb-associated endophthalmitis (BAE), the majority of reported cases, $53-82 \%$, received adjunctive IVD [9-12]. No BAE series however has yet reported the VA outcomes of cases treated with and without IVD. Unlike postcataract endophthalmitis, BAE is commonly associated with virulent Streptococcus and Gram-negative organisms [10-13]. If IVD potentiates intraocular infection then VA outcomes may be worse in BAE cases treated with IVD. Additionally, the culture data of second biopsies in BAE cases treated with IVD may manifest a higher rate of persistent infection. The current study reports the VA outcomes and culture results of BAE cases treated with and without IVD to further clarify the role IVD plays in the management of bacterial endophthalmitis.

\section{Methods}

The study protocol was approved by the Institutional Review Board of the University of Miami Miller School of Medicine Subcommittee for the Protection of Human Subjects in Research. The medical records and microbiologic records of all patients treated for BAE at Bascom Palmer Eye Institute (BPEI) between January 1, 1996 and December 31, 2009 were reviewed. As a nonrandomized comparative case series the decision to use or not use IVD was made by the individual 


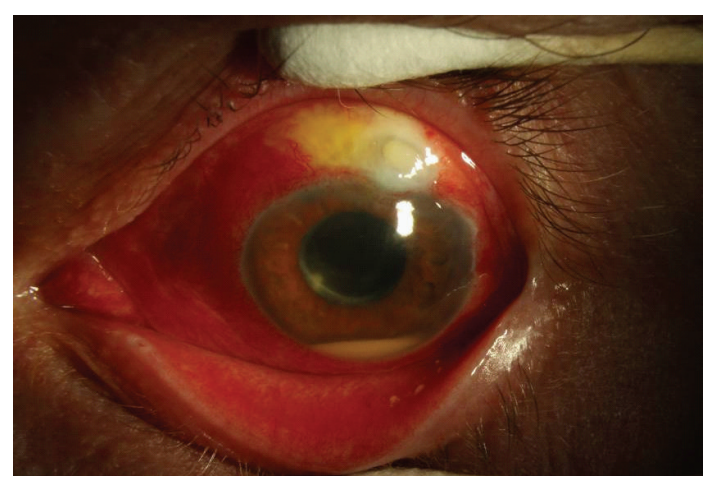

(a)

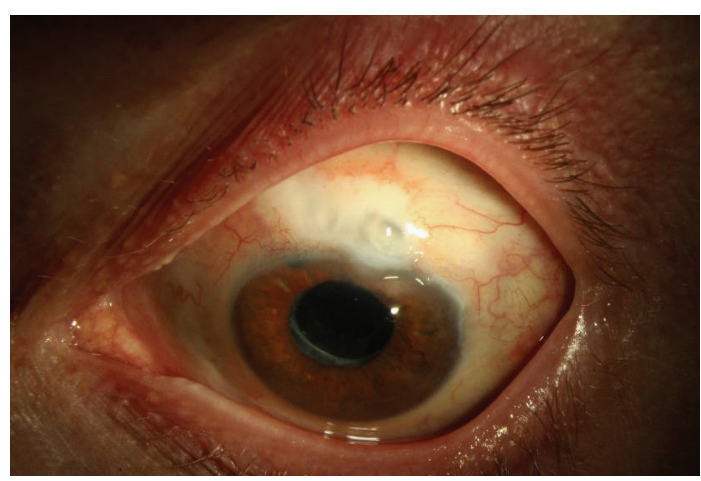

(b)

FIGURe 1: Photographs of the left eye of 55-year-old male presenting with BAE from Moraxella. (a) Presenting VA: HM, IOP: 19 mmHg. Treatment: pars plana vitrectomy with intravitreal Vancomycin, Ceftazidime, and Dexamethasone. (b) At 3 months VA: 20/40, IOP: $14 \mathrm{mmHg}$.

treating physician and did not involve a prospective protocol. All patients had prior glaucoma filtering surgery. BAE was defined as intraocular infection with vitreous involvement receiving treatment with intravitreal antibiotics. Patients with tube shunts as the filtering mechanism, bleb infection only (no posterior inflammation), onset within 1 month of glaucoma surgery, and inadvertent filtering blebs after cataract surgery were excluded. Clinical history and presentation, treatment, intraocular culture data, VA outcomes, and factors affecting VA were recorded. The current study included clinical information from the BPEI series of BAE previously published $[9,13]$.

Snellen VAs were converted to $\log$ MAR equivalents for statistical analysis; VAs of HM, LP, and NLP were assigned $\operatorname{logMAR}$ values of $2.6,3$, and 4 respectively. Change in VA was determined by comparing the last recorded VA before the onset of endophthalmitis (pre-endophthalmitis VA) with VA at 1 and 3 months. Three or more lines of improvement ( $\geq 3$ lines improvement) were determined by comparing the VA at presentation of endophthalmitis (presentation VA) with the VA at 1 and 3 months. The mean logMAR change after presentation $(\log M A R \Delta)$ and other clinical data were grouped according to IVD use and compared using the 2 -sided Student's $t$-test. Logistic regression was used to determine the odds ratio for IVD as a predictive factor for $\geq 3$ lines improvement. A $P$ value of $\leq 0.05$ was considered statistically significant.

\section{Results}

In the current study, 86 eyes were identified. Excluded were 1 eye with a preexisting tube shunt and 2 eyes that underwent primary evisceration. Of the 83 eyes, $70(84 \%)$ received IVD and $13(16 \%)$ did not receive IVD. None of the patients received systemic steroid. In all cases, the causative organisms were sensitive to the intravitreal antibiotics clinically administered. Baseline demographics, clinical presentation, and initial culture results were similar between the two groups with a few exceptions (Table 1).
A greater percentage of IVD cases presented with poor view of the fundus, $69 \%$ compared to $39 \%$. The majority of both groups received an initial treatment of tap and injection (T\&I); however, a higher percentage received pars plana vitrectomy (PPV) in the IVD group, $41 \%$ compared to $8 \%$. Also a higher percentage of IVD cases were culture-positive, $66 \%$ compared to $46 \%$, but this difference did not reach significance.

Repeat cultures were performed during a second procedure in $11 / 70(16 \%)$ IVD cases and $3 / 13(23 \%)$ of cases that did not receive IVD (Table 2).There was no significant difference in primary or repeat culture-positive results between the two groups. The repeat culture-positive rate was $2 / 70(3 \%)$ for IVD cases and $1 / 13(8 \%)$ for the cases that did not receive IVD. In each of these 3 cases the same causative organism was isolated in the repeat culture as in the initial culture. Repeat cultures were positive in $1 / 21$ (5\%) Streptococcus and 2/6 (33\%) Enterococcus cases.

IVD cases had worse mean pre-endophthalmitis and presentation VA but this did not reach significance (Table 3 ).

At 1 month mean VA was 5/200 in the IVD group and 1.8/ 200 in the group that did not receive IVD, $\log$ MAR $\Delta$ of 0.85 and 1.56 , respectively $(P=0.02)$. At 3 months mean VA was $7 / 200$ in the IVD group and $3 / 200$ in the group that did not receive IVD, $\operatorname{logMAR} \Delta$ of 0.74 and 1.33 , respectively $(P=0.14)$. A higher percentage of IVD cases achieved $\geq 3$ lines improvement at 1 and 3 months. Logistic regression showed that IVD was a significant predictive factor of $\geq 3$ lines improvement at both 1 and 3 months (Table 4).

\section{Discussion}

Corticosteroids are often used as an important adjunct to antibiotics and PPV in the management of infectious bacterial endophthalmitis (Figure 1). Corticosteroids are known to reduce the degree of inflammation caused by toxins liberated from microorganisms. The role of IVD in the management of postcataract bacterial endophthalmitis is unclear due to contradictory results of small, comparative studies (Table 5). 
TABle 1: Baseline demographics, clinical presentation, and initial culture results.

\begin{tabular}{|c|c|c|c|}
\hline & $\begin{array}{c}\text { IVD } \\
70 / 83(84 \%)\end{array}$ & $\begin{array}{c}\text { No IVD } \\
13 / 83(16 \%)\end{array}$ & $P$ value \\
\hline \multicolumn{4}{|l|}{ Age } \\
\hline Mean, SD & 74 yr (12) & 70 yr (14) & 0.27 \\
\hline \multicolumn{4}{|l|}{ Gender } \\
\hline Female & $34(49 \%)$ & $8(62 \%)$ & 0.39 \\
\hline Male & $36(51 \%)$ & $5(39 \%)$ & \\
\hline \multicolumn{4}{|l|}{ Diabetes mellitus } \\
\hline Present & $9(13 \%)$ & $2(15 \%)$ & 0.81 \\
\hline Absent & $61(87 \%)$ & $11(85 \%)$ & \\
\hline \multicolumn{4}{|l|}{$\begin{array}{l}\text { Antimetabolites } \\
\text { (MMC or 5FU) }\end{array}$} \\
\hline Used & $45(64 \%)$ & $7(54 \%)$ & 0.47 \\
\hline Not used & $25(36 \%)$ & $6(46 \%)$ & \\
\hline Mean time of onset, SD & 60 mo (43) & 49 mo (55) & 0.46 \\
\hline \multicolumn{4}{|l|}{ Bleb leak } \\
\hline Present & $16(23 \%)$ & $5(38 \%)$ & 0.23 \\
\hline Absent & $54(77 \%)$ & $8(62 \%)$ & \\
\hline \multicolumn{4}{|l|}{ Anterior chamber } \\
\hline Hypopyon & $48(69 \%)$ & $10(77 \%)$ & 0.55 \\
\hline \multicolumn{4}{|l|}{ View to fundus } \\
\hline Hazy & $22(31 \%)$ & $8(62 \%)$ & 0.04 \\
\hline Poor/none & $48(69 \%)$ & $5(39 \%)$ & \\
\hline \multicolumn{4}{|l|}{ Intraocular Pressure } \\
\hline Presentation, SD & $20(14)$ & $19(12)$ & 0.8 \\
\hline \multicolumn{4}{|l|}{ Treatment, initial } \\
\hline Tap and injection & $41(59 \%)$ & $12(92 \%)$ & 0.03 \\
\hline Pars plana vitrectomy & $29(41 \%)$ & $1(8 \%)$ & \\
\hline \multicolumn{4}{|l|}{ Treatment, additional } \\
\hline Filtering procedure & $12(17 \%)$ & $1(8 \%)$ & 0.39 \\
\hline Pars plana vitrectomy & $21(30 \%)$ & $2(15 \%)$ & 0.28 \\
\hline \multicolumn{4}{|l|}{ Culture results } \\
\hline Culture positive & $46(66 \%)$ & $6(46 \%)$ & 0.18 \\
\hline Culture negative & $24(34 \%)$ & $7(54 \%)$ & \\
\hline Gram-positive cases & $33(47 \%)$ & $4(31 \%)$ & 0.28 \\
\hline Streptococcus & $19(27 \%)$ & $2(15 \%)$ & 0.37 \\
\hline $\begin{array}{l}\text { Coagulase-negative } \\
\text { Staphylococcus }\end{array}$ & $7(10 \%)$ & $2(15 \%)$ & 0.57 \\
\hline Enterococcus & $6(9 \%)$ & 0 & 0.27 \\
\hline Staphylococcus aureus & $1(1 \%)$ & 0 & 0.67 \\
\hline Gram-negative cases & $12(17 \%)$ & $2(15 \%)$ & 0.88 \\
\hline Moraxella & $8(11 \%)$ & 0 & 0.2 \\
\hline Pseudomonas & $2(3 \%)$ & $1(8 \%)$ & 0.39 \\
\hline Serratia & $1(1 \%)$ & $1(8 \%)$ & 0.18 \\
\hline
\end{tabular}

Das et al. reported favorable results of reduced intraocular inflammation at 1 and 4 weeks in eyes treated with IVD [5]. Gan et al. additionally found a trend toward better visual outcomes at 3 and 12 months in eyes treated with IVD [6]. In contrast, Hall et al. reported no difference in inflammation
TABLE 2: Repeat culture results.

\begin{tabular}{lccc}
\hline & IVD & No IVD & $P$ value \\
& $70 / 83$ & $13 / 83$ & \\
& $(84 \%)$ & $(16 \%)$ & \\
\hline Repeat Cultures Performed: & & & \\
Number of eyes & $11(16 \%)$ & $3(23 \%)$ & 0.52 \\
Mean time, days (range) & $20(1-60)$ & $14(2-30)$ & 0.64 \\
Primary culture results & & & \\
Streptococcus & $4(36 \%)$ & $2(67 \%)$ & \\
Enterococcus faecalis & $2(18 \%)$ & 0 & \\
Coagulase-neg Staph. & $2(18 \%)$ & $1(33 \%)$ & \\
Enterobacter aerogenes & $1(9 \%)$ & 0 & 0.43 \\
No growth & $2(18 \%)$ & 0 & \\
Repeat culture results & & & \\
Streptococcus & 0 & $1(33 \%)$ & \\
Enterococcus faecalis & $2(18 \%)$ & 0 & \\
Coagulase-neg Staph. & 0 & 0 & 0.57 \\
Enterobacter aerogenes & 0 & 0 & \\
No growth & $9(82 \%)$ & $2(67 \%)$ & \\
\hline Repeat culture positive rate & $2(3 \%)$ & $1(8 \%)$ & 0.57 \\
\hline
\end{tabular}

TABLE 3: VA outcomes.

\begin{tabular}{|c|c|c|c|}
\hline & $\begin{array}{c}\text { IVD } \\
71 / 84(84 \%) \\
\end{array}$ & $\begin{array}{c}\text { No IVD } \\
13 / 84(16 \%) \\
\end{array}$ & $P$ value \\
\hline $\begin{array}{l}\text { VA, pre- } \\
\text { endophthalmitis }\end{array}$ & $n=67$ & $n=13$ & \\
\hline Mean & $20 / 90$ & $20 / 70$ & 0.57 \\
\hline Range & 20/20-LP & $20 / 25-20 / 400$ & \\
\hline VA, presentation & $n=70$ & $n=13$ & \\
\hline Mean & $0.9 / 200$ & $1.7 / 200$ & 0.23 \\
\hline Range & 20/40-NLP & 20/80-LP & \\
\hline VA, 1 month & $n=66$ & $n=12$ & \\
\hline Mean & $5 / 200$ & $1.8 / 200$ & 0.14 \\
\hline Range & 20/25-NLP & 20/25-NLP & \\
\hline $\begin{array}{l}\geq 3 \text { lines } \\
\text { Improvement }\end{array}$ & $44(67 \%)$ & $3(25 \%)$ & 0.01 \\
\hline $\log$ MAR $\Delta$ & 0.85 & 1.56 & 0.02 \\
\hline VA, 3 months & $n=56$ & $n=9$ & \\
\hline Mean & $7 / 200$ & $3 / 200$ & 0.36 \\
\hline Range & 20/25-NLP & 20/25-LP & \\
\hline $\begin{array}{l}\geq 3 \text { lines } \\
\text { Improvement }\end{array}$ & $36(64 \%)$ & $3(33 \%)$ & 0.14 \\
\hline $\log$ MAR $\Delta$ & 0.74 & 1.33 & 0.14 \\
\hline
\end{tabular}

Table 4: Predictive factor $\geq 3$ lines improvement.

\begin{tabular}{lcc}
\hline IVD versus No IVD & Odds ratio, $(\mathrm{CI})$ & $P$ value \\
\hline 1 month & $7.04(1.63,30.43)$ & 0.01 \\
3 months & $5.21(1.07,25.37)$ & 0.04 \\
\hline
\end{tabular}

and VA outcomes at last followup in eyes treated with IVD [7]. Shah et al. found worse VA outcomes at 1, 3, and 6 
TABLE 5: Comparative studies of IVD for bacterial endophthalmitis.

\begin{tabular}{|c|c|c|c|c|c|c|c|}
\hline & $\begin{array}{c}\text { Clinical setting } \\
n\end{array}$ & Staph epi. & $\begin{array}{c}\text { Culture results } \\
\text { Strep/Enterococcus }\end{array}$ & Gram-negative & Inflammation & VA outcomes & Time \\
\hline Das et al. [5] & $\begin{array}{c}\text { Postcataract and } \\
\text { trauma }\end{array}$ & & & & & & \\
\hline IVD & 29 & $\mathrm{n} / \mathrm{a}$ & $\mathrm{n} / \mathrm{a}$ & $\mathrm{n} / \mathrm{a}$ & 2.6 score & $86 \%$ success & \multirow{2}{*}{3 months } \\
\hline No IVD & 34 & & & & 3.2 score $^{1}$ & $71 \%$ success & \\
\hline Shah et al. [8] & Postcataract & & & & \multirow{3}{*}{$\mathrm{n} / \mathrm{a}$} & & \multirow{3}{*}{6 months } \\
\hline IVD & 26 & $31 \%$ & $12 \%$ & 0 & & 20/70 median & \\
\hline No IVD & 31 & $35 \%$ & $13 \%$ & $3 \%$ & & 20/50 median $^{2}$ & \\
\hline Gan et al. [6] & Postcataract & & & & \multirow{3}{*}{$\mathrm{n} / \mathrm{a}$} & & \\
\hline IVD & 16 & $39 \%$ & $8 \%$ & 0 & & $85 \% 20 / 200$ or better & \multirow{2}{*}{3 months } \\
\hline No IVD & 13 & $50 \%$ & $6 \%$ & 0 & & $50 \% 20 / 200$ or better $^{3}$ & \\
\hline Hall et al. [7] & Postcataract & & & & & & \multirow{3}{*}{$\begin{array}{l}\text { last } \\
\text { followup }\end{array}$} \\
\hline IVD & 26 & $46 \%$ & $23 \%$ & 0 & 0.3 cell/flare & 20/40 median & \\
\hline No IVD & 38 & $37 \%$ & $5 \%$ & 0 & 0.3 cell/flare & 20/50 median & \\
\hline Jacobs et al. & Bleb-associated & & & & \multirow{3}{*}{$\mathrm{n} / \mathrm{a}$} & & \multirow{3}{*}{3 months } \\
\hline IVD & 70 & $10 \%$ & $36 \%$ & $17 \%$ & & 7/200 mean & \\
\hline No IVD & 13 & $15 \%$ & $15 \%$ & $15 \%$ & & $3 / 200$ mean $^{4}$ & \\
\hline
\end{tabular}

${ }^{1}$ Relative change in inflammation showed statistical significance at 1 and 4 weeks, not at 3 months. ${ }^{2} P<0.05,{ }^{3} P=0.055 .{ }^{4}$ Relative logMAR showed statistical significance at 1 month, not at 3 months.

TABLE 6: PPV versus T\&I in present BAE series.

\begin{tabular}{lccc}
\hline & PPV & T\&I & $P$ value \\
& $29 / 83(35 \%)$ & $54 / 83(65 \%)$ & \\
\hline VA, pre- & $n=28$ & $n=53$ & \\
endophthalmitis & $20 / 55$ & $20 / 50$ & 0.3 \\
Mean & $20 / 20-\mathrm{CF}$ & $20 / 20-\mathrm{LP}$ & \\
Range & $n=30$ & $n=54$ & \\
VA, presentation & $\mathrm{LP}$ & $\mathrm{HM}$ & 0.02 \\
Mean & $20 / 80-\mathrm{LP}$ & $20 / 40-\mathrm{NLP}$ & \\
Range & $n=27$ & $n=39$ & \\
VA, 3 months & $3 / 200$ & $20 / 390$ & \\
Mean & $20 / 25-\mathrm{NLP}$ & $20 / 25-\mathrm{LP}$ & \\
Range & 1.23 & 0.57 & 0.02 \\
logMAR $\Delta$ & & & \\
\hline
\end{tabular}

months in eyes treated with IVD [8]. The present series was unique as it was the first to study BAE cases that had a higher rate of Streptococcus and Gram-negative cases.

BAE studies are limited by the relatively small number of BAE cases. Conclusions in BAE studies are found in the inherent limitations of retrospective nonrandomized data. The majority of cases in the present study received IVD which is similar to other BAE series [9-12]. Overall the two groups compared in this study had similar baseline demographic, clinical presentation, and initial culture data (Table 1).

A difference was found in the initial treatment of the two groups. The majority of cases in both groups received T\&I as the initial treatment, however the percentage that received initial PPV was higher in the IVD group. The effect this
TABLE 7: PPV versus T\&I: presentation VA of LP or worse in present BAE series.

\begin{tabular}{lccc}
\hline & PPV & T\&I & $P$ value \\
& $18 / 26(69 \%)$ & $8 / 26(31 \%)$ & \\
\hline VA, pre- & $n=18$ & $n=8$ & \\
endophthalmitis & $20 / 65$ & $20 / 270$ & 0.16 \\
$\quad$ Mean & $20 / 20-1 / 200$ & $20 / 25-\mathrm{HM}$ & \\
Range & $n=18$ & $n=8$ & \\
VA, presentation & LP & LP & 0.35 \\
Mean & LP & LP-NLP & \\
Range & $n=17$ & $n=5$ & \\
VA, 3 months & $1 / 200$ & $1 / 200$ & \\
Mean & $20 / 60-\mathrm{NLP}$ & $20 / 200-\mathrm{LP}$ & \\
Range & 1.71 & 1.18 & 0.46 \\
logMAR $\Delta$ & &
\end{tabular}

difference had on the VA outcomes is unclear. A comparison of PPV and T\&I cases in this series showed that PPV cases had significantly worse mean VA at presentation and 3 months (Table 6).

When presentation of VA was LP or worse, 3-month$\log$ MAR $\triangle$ was worse in the PPV group but not significantly (Table 7).

As VA outcomes with PPV were worse than T\&I in this series, it is unlikely that improved VA outcomes in the IVD group were due to a higher percentage of initial treatment with PPV.

The repeat culture results in this series were similar to the Endophthalmitis Vitrectomy Study (EVS). In the EVS, 14 of $420(3.3 \%)$ had positive repeat cultures [14]. In the present 
series the overall repeat culture-positive rate was 3 of 83 (3.6\%). Of note the EVS did not use IVD and the EVS had a lower rate of Streptococcus and Gram-negative organisms, yet the rate of persistent infection was similar in the EVS to the present study. The present study confirms the observation made by Shaarawy et al. that persistent infection can occur in bacterial endophthalmitis and appears to be more common with virulent organisms such as Streptococcus and Enterococcus [15]. Although persistent infection occurred in the present BAE series there was not a higher rate among the cases treated with IVD.

VA outcomes in the present series confirm the clinical observation by Irvine et al. that intraocular steroids appeared to hasten visual recovery [2]. At 1 month, $67 \%$ gained $\geq 3$ lines in the IVD group compared to $25 \%$ in the group that did not receive IVD. The VA gains in the IVD group were more significant at 1 month than 3 months. Logistic regression did show that IVD was a predictive factor of $\geq 3$ lines of improvement at both 1 and 3 months. VA gains may have been due to a decrease in intraocular inflammation, but a standardized manner of grading inflammation was not employed in the present series.

Limitations of the current study include the retrospective nature, small sample size in the control arm, and lack of a definitive treatment protocol. This study does demonstrate that IVD was associated with improved short-term VA outcomes and did not potentiate infection in BAE.

\section{Disclosure}

The authors in this paper have no financial or proprietary interests in products, methods, or materials published in this paper.

\section{Acknowledgment}

This paper was supported by the National Institute of Health Center Grant P30-EY014801, an unrestricted grant to the University of Miami from Research to Prevent Blindness, New York, NY, USA.

\section{References}

[1] G. A. Peyman and R. Herbst, "Bacterial endophthalmitis: treatment with intraocular injection of gentamicin and dex amethasone," Archives of Ophthalmology, vol. 91, no. 5, pp. 416-418, 1974.

[2] W. D. Irvine, H. W. Flynn, D. Miller, and S. C. Pflugfelder, "Endophthalmitis caused by gram-negative organisms," Archives of Ophthalmology, vol. 110, no. 10, pp. 1450-1454, 1992.

[3] J. A. Schulman and G. A. Peyman, "Intravitreal corticosteroids as an adjunct in the treatment of bacterial and fungal endophthalmitis: a review," Retina, vol. 12, no. 4, pp. 336-340, 1992.

[4] J. S. Pollack, "ASRS pat survey," 2004, http://www.asrs.org/.

[5] T. Das, S. Jalali, V. K. Gothwal, S. Sharrna, and T. J. Naduvilath, "Intravitreal dexamethasone in exogenous bacterial endophthalmitis: results of a prospective randomised study," British Journal of Ophthalmology, vol. 83, no. 9, pp. 1050-1055, 1999.

[6] I. M. Gan, L. C. Ugahary, J. T. van Dissel et al., "Intravitreal dexamethasone as adjuvant in the treatment of postoperative endophthalmitis: a prospective randomized trial," Graefe's Archive for Clinical and Experimental Ophthalmology, vol. 243, no. 12, pp. 1200-1205, 2005.

[7] E. F. Hall, G. R. Scott, D. C. Musch, and D. N. Zacks, "Adjunctive intravitreal dexamethasone in the treatment of acute endophthalmitis following cataract surgery," Journal of Clinical Ophthalmology, vol. 2, no. 1, pp. 139-145, 2008.

[8] G. K. Shah, J. D. Stein, S. Sharma et al., "Visual outcomes following the use of intravitreal steroids in the treatment of postoperative endophthalmitis," Ophthalmology, vol. 107, no. 3, pp. 486-489, 2000.

[9] T. Leng, D. Miller, H. W. Flynn, D. J. Jacobs, and S. J. Gedde, "Delayed-onset bleb-associated endophthalmitis (19962008): causative organisms and visual acuity outcomes," Retina, vol. 31, no. 2, pp. 344-352, 2010.

[10] T. A. Kangas, D. S. Greenfield, H. W. Flynn, R. K. Parrish, and P. Palmberg, "Delayed-onset endophthalmitis associated with conjunctival filtering blebs," Ophthalmology, vol. 104, no. 5, pp. 746-752, 1997.

[11] B. G. Busbee, F. M. Recchia, R. Kaiser et al., "Bleb-associated endophthalmitis: clinical characteristics and visual outcomes," Ophthalmology, vol. 111, no. 8, pp. 1495-1503, 2004.

[12] S. Mandelbaum, R. K. Forster, H. Gelender, and W. Culbertson, "Late onset endophthalmitis associated with filtering blebs," Ophthalmology, vol. 92, no. 7, pp. 964-972, 1985.

[13] D. J. Jacobs, T. Leng, H. W. Flynn Jr., W. Shi, D. Miller, and S. J. Gedde, "Delayed-onset bleb-associated endophthalmitis: presentation and outcome by culture result," Clinical Ophthalmology, vol. 5, no. 1, pp. 739-744, 2011.

[14] B. H. Doft, S. F. Kelsey, and S. R. Wisniewski, "Additional procedures after the initial vitrectomy or tap-biopsy in the endophthalmitis vitrectomy study," Ophthalmology, vol. 105, no. 4, pp. 707-716, 1998.

[15] A. Shaarawy, M. G. Grand, T. A. Meredith, and H. E. Ibanez, "Persistent endophthalmitis after intravitreal antimicrobial therapy," Ophthalmology, vol. 102, no. 3, pp. 382-387, 1995. 


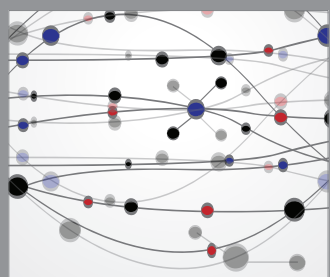

The Scientific World Journal
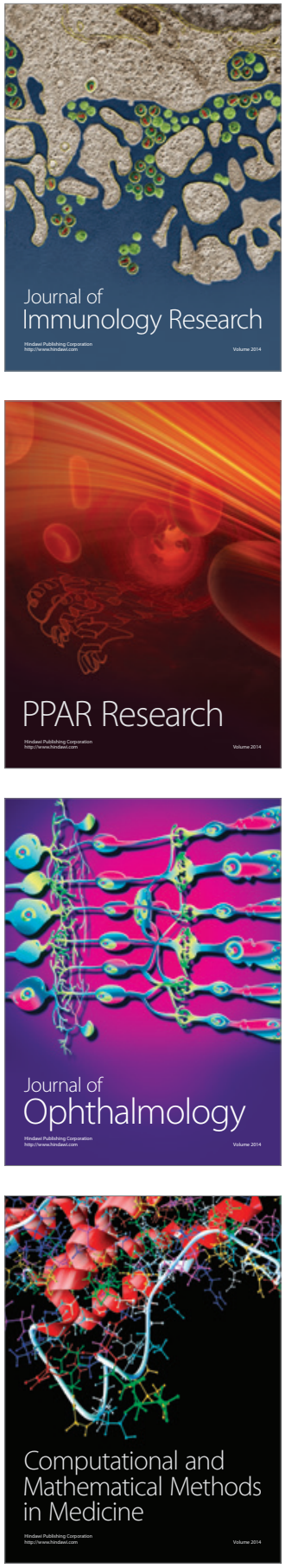

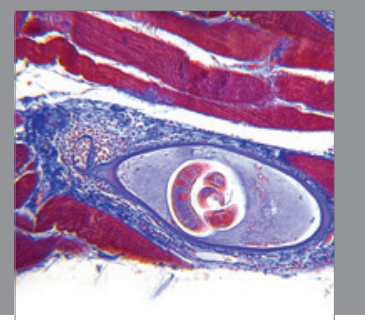

Gastroenterology

Research and Practice
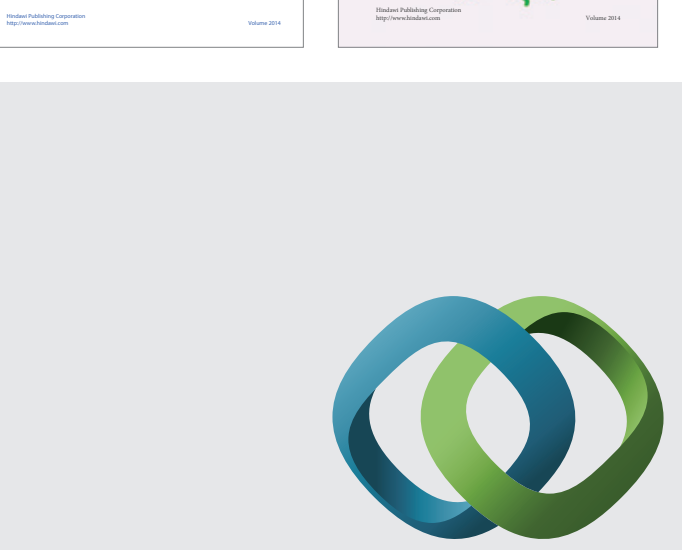

\section{Hindawi}

Submit your manuscripts at

http://www.hindawi.com
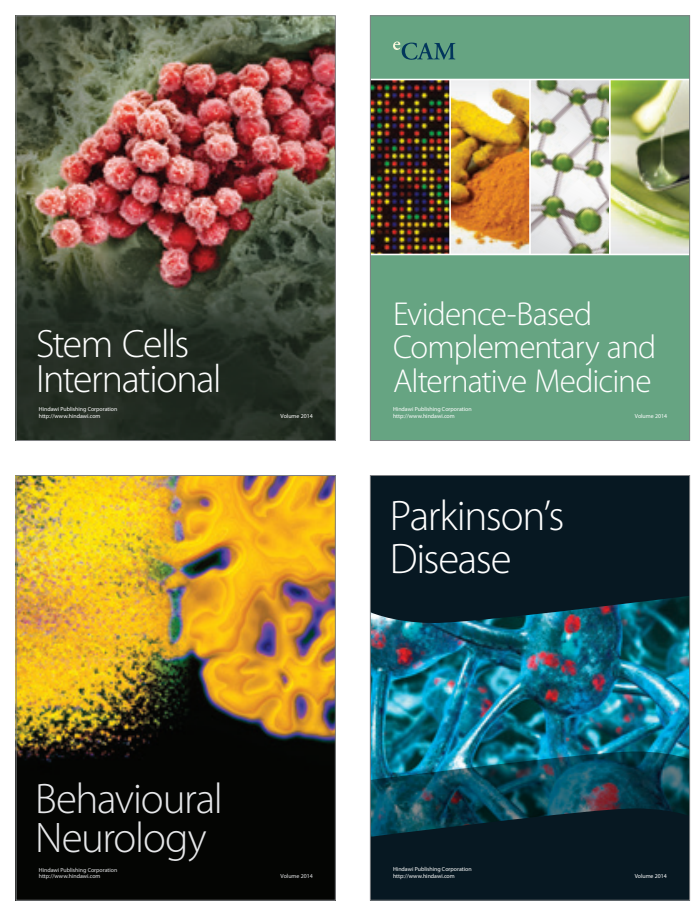

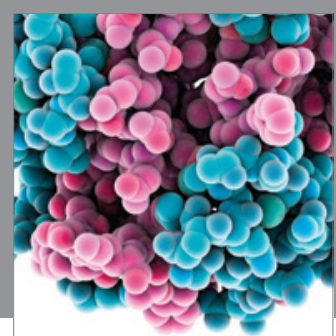

Journal of
Diabetes Research

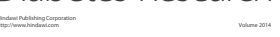

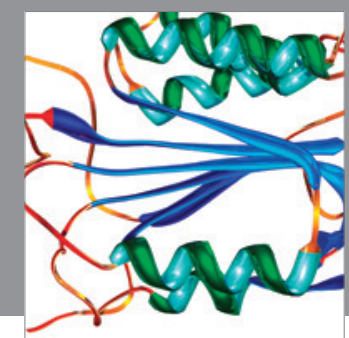

Disease Markers
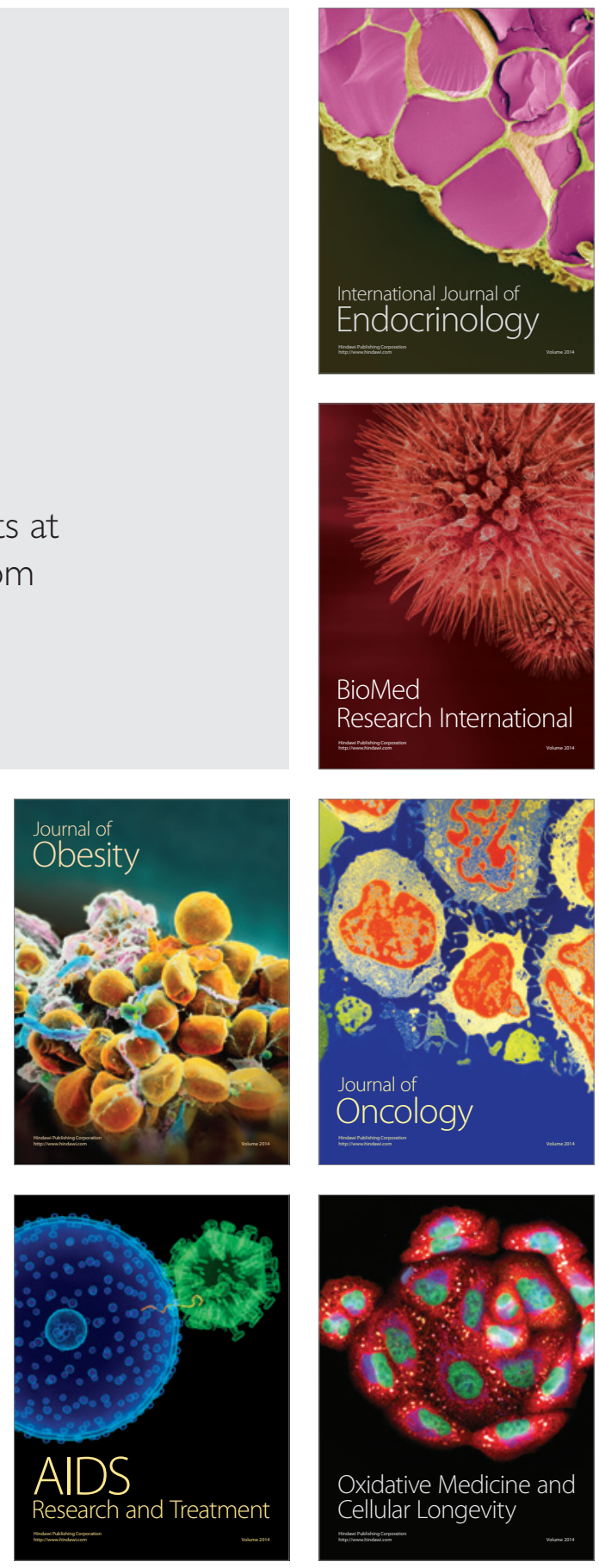